\title{
CLUSTER CONTROL
}

Protein therapeutics could benefit from using highly concentrated solutions, which would enable high doses to be delivered without the need for the long, slow intravenous drip that lower concentrations require. But it's not easy to prepare such potent solutions. For one thing, soluble proteins develop high viscosities at high concentrations, making injection impossible. Worse, concentrated proteins tend to aggregate and gel, precipitating as a sticky, nonfunctional mess.

Yet concentrated solutions of functional proteins are evidently possible, because they occur in cells, where macromolecules may constitute up to 40 per cent of the volume. One reason why aggregation is not, in general, a problem here is that proteins are kept in compact, soluble conformations by molecular crowding. The proximity of other molecules biases the equilibrium towards the fully folded state, because any tendency to unfold would exclude these solutes from more of the available volume, which is entropically unfavourable.

The stability of the cytoplasm is thus a very delicately balanced compromise between several competing influences with different intrinsic length scales: crowding, aggregation and electrostatic interactions between charged proteins. Johnston et al. have now shown that, by considering such a system as a colloidal dispersion in which the various interactions can be chemically tuned, it is possible to make highly concentrated, low-viscosity solutions of biologically viable proteins selfassembled into nanoscale clusters ${ }^{1}$.

To form these clusters, protein monomers must be made attractive to each other while remaining folded. This is achieved by adding a cosolvent, the sugar trehalose (used by some organisms as a cryoprotectant), which creates an entropic attraction due to excluded-volume effects: protein association increases the volume accessible to the cosolvent. By reducing electrostatic repulsion between proteins with a $\mathrm{pH}$ buffer that adjusts the surface charge, this attraction can be made dominant.

If the clusters grew indefinitely, they would eventually just precipitate. But their accumulating charge eventually counteracts the attachment of new monomers. The size at which this happens depends on the trehalose concentration and the $\mathrm{pH}$, and can be adjusted between about 50 and $300 \mathrm{~nm}$. Meanwhile, the clusters' net charge ensures that they repel one another.

Crucially, the clustered proteins remain stable and functional when dilution disperses them, apparently because self-crowding protects against unfolding. Chromatographic measurements reveal no significant increase in high-molecular-weight aggregates in the diluted solutions.

The clusters' nanoscale size maintains a healthily low viscosity, suitable for injection. Subcutaneous

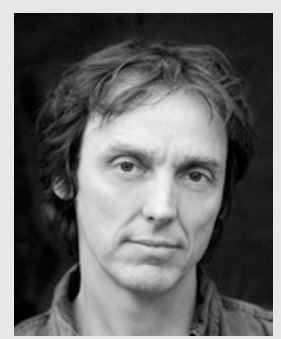

PHILIP BALL

injection of nanoclusters of toxin-binding antibodies in mice results in pharmacological effects indistinguishable from those of an equivalent dose administered in low-concentration solutions. Because the concepts of colloidal control used here are quite general, there is good reason to expect the approach to work for other proteins. Quite aside from the important practical implications, the results offer a reminder that living cells are not just bags of chemicals. Small and nanoscale clusters have been observed before in concentrated protein solutions, sometimes in a dynamic, metastable state ${ }^{2,3}$, and it seems that cells might be best regarded as a highly heterogeneous colloidal mixture operating on the exquisitely tuned edge of physicochemical stability.

References

1. Johnston, K. P. et al. ACS Nano http://dx.doi.org/ $10.1021 / \mathrm{nn} 204166 \mathrm{z}$ (2012).

2. Stradner, A. et al. Nature 432, 492-495 (2004).

3. Gliko, O. et al. J. Phys. Chem. B 111, 3106-3114 (2007).

\section{Dimensions are critical}

\section{Results from a cubic heavy-fermion compound provide a new perspective on quantum criticality in these types of material.}

\section{Piers Coleman}

$\mathrm{n}$ today's ongoing quest to synthesize solid-state materials exhibiting intriguing quantum mechanical properties, a promising guiding principle is that new phases occur at the edge of a magnetic instability ${ }^{1,2}$. The ground state of a metal becomes unstable to magnetism at a zero-temperature quantum phase transition. If the transition is not abrupt but smooth, the point in the phase diagram of the material in which different phases meet is called a quantum critical point (QCP). Around the QCP, the properties of the electron fluids exhibit large fluctuations, which can have dramatic consequences and in some cases completely transform the character of a metal. In fact, QCPs seem to form vital nucleating points for new phases of matter and types of material behaviour ${ }^{2}$.

Elucidating the physics of QCPs is therefore essential to understanding the 